\title{
Development and Validation of a Mucosal Antibody (IgA) Test to Identify Persistent Infection with Foot-and-Mouth Disease Virus
}

\author{
Jitendra K. Biswal (D, Antonello Di Nardo, Geraldine Taylor, David J. Paton and Satya Parida *(D) \\ The Pirbright Institute, Ash Road, Pirbright, Surrey GU24 0NF, UK; jkubiswal@gmail.com (J.K.B.); \\ antonello.dinardo@pirbright.ac.uk (A.D.N.); geraldine.taylor@pirbright.ac.uk (G.T.); \\ david.paton@pirbright.ac.uk (D.J.P.) \\ * Correspondence: satyaparida1964@gmail.com; Tel.: +44-7920512797
}

check for

updates

Citation: Biswal, J.K.; Nardo, A.D.;

Taylor, G.; Paton, D.J.; Parida, S.

Development and Validation of a

Mucosal Antibody (IgA) Test to

Identify Persistent Infection with

Foot-and-Mouth Disease Virus.

Viruses 2021, 13, 814. https:/ /

doi.org/10.3390/v13050814

Academic Editor: Halle

Bielefeldt-Ohmann

Received: 31 March 2021

Accepted: 28 April 2021

Published: 1 May 2021

Publisher's Note: MDPI stays neutral with regard to jurisdictional claims in published maps and institutional affiliations.

Copyright: (C) 2021 by the authors. Licensee MDPI, Basel, Switzerland. This article is an open access article distributed under the terms and conditions of the Creative Commons Attribution (CC BY) license (https:/ / creativecommons.org/licenses/by/ $4.0 /)$.
Abstract: It is well known that approximately 50\% of cattle infected with foot-and-mouth disease (FMD) virus (FMDV) may become asymptomatic carrier (persistently infected) animals. Although transmission of FMDV from carrier cattle to naïve cattle has not been demonstrated experimentally, circumstantial evidence from field studies has linked FMDV-carrier cattle to cause subsequent outbreaks. Therefore, the asymptomatic carrier state complicates the control and eradication of FMD. Current serological diagnosis using tests for antibodies to the viral non-structural proteins (NSP-ELISA) are not sensitive enough to detect all carrier animals, if persistently infected after vaccination and do not distinguish between carriers and non-carriers. The specificity of the NSP ELISA may also be reduced after vaccination, in particular after multiple vaccination. FMDV-specific mucosal antibodies (IgA) are not produced in vaccinated cattle but are elevated transiently during the acute phase of infection and can be detected at a high level in cattle persistently infected with FMDV, irrespective of their vaccination status. Therefore, detection of IgA by ELISA may be considered a diagnostic alternative to RT-PCR for assessing FMDV persistent infection in ruminants in both vaccinated and unvaccinated infected populations. This study reports on the development and validation of a new mucosal IgA ELISA for the detection of carrier animals using nasal, saliva, and oro-pharyngeal fluid (OPF) samples. The diagnostic performance of the IgA ELISA using nasal samples from experimentally vaccinated and infected cattle demonstrated a high level of specificity (99\%) and an improved level of sensitivity (76.5\%). Furthermore, the detection of carrier animals reached $96.9 \%$ when parallel testing of samples was carried out using both the IgA-ELISA and NSP-ELISA.

Keywords: foot-and-mouth disease; ELISA; mucosal IgA; carrier (persistent infection); post-outbreak surveillance; nasal; saliva and oro-pharyngeal fluid (OPF)

\section{Introduction}

Foot-and-mouth disease (FMD) is a highly contagious vesicular disease caused by FMD virus (FMDV), an aphthovirus within the family picornaviridae that infects both domesticated and wild cloven-hoofed animals [1]. The classical FMD symptoms in ruminants are characterised by fever, inappetence, lameness, excess salivation and vesicles in and around the mouth, teats and feet. These clinical signs normally subside approximately 10-14 days post-infection [2]. However, up to 50\% of FMD-recovered cattle may harbour virus in their oro-pharyngeal and naso-pharyngeal cavity at 28 or more days post-infection and are known as FMDV-carriers or persistently infected animals [3,4]. This asymptomatic carrier state of FMD complicates the control and eradication of the disease. The duration of the FMDV-carrier state may be influenced by a combination of viral and host-factors, and can last from months to years [5]. Although transmission of FMD virus from domestic animal carriers to susceptible naïve animals has not been demonstrated under experimental 
conditions [6,7], circumstantial evidence from field studies has linked FMDV-carrier cattle to subsequent outbreaks [8-10]. Furthermore, a recent experimental study has demonstrated clinical infection in naïve cattle when inoculated with oro-pharyngeal fluids (OPF) obtained from carrier animals [11]. Since FMDV-carriers may be considered a risk for transmitting infection, they must be identified by post-vaccination serosurveillance to substantiate freedom from infection to regain the "FMD-free status without vaccination" for the purpose of international trade [12,13]. Carrier animals persistently infected with FMDV can be identified by detection of the virus in OPF collected with a probang sampling cup. However, recovery of infectious virus or viral genome from such oro-pharyngeal scrapings of FMDV persistently infected cattle is intermittent [12,14].

Tests for the detection of antibodies to FMDV non-structural proteins (NSP) have been used for detection of infection in vaccinated animals (DIVA). However, the currently validated NSP antibody tests [15] may not detect all infected animals within a vaccinated population [16] and do not distinguish between carriers and those that have eliminated FMDV. Therefore, new NSP tests or alternative tests which can be used either as screening tests or confirmatory tests to the existing NSP tests are needed.

Several studies describe the presence of FMDV-specific mucosal $\operatorname{IgA}(\operatorname{IgA})$ in oropharyngeal fluid as an indicator of FMDV persistence [14,17-19]. An IgA-ELISA, using saliva samples, to detect FMD carrier cattle following vaccination and challenge exposure has been developed previously [14]. Although this IgA-ELISA can detect carrier cattle in vaccinated and unvaccinated populations, the non-specific reactions with some saliva samples from uninfected animals have hampered the introduction of the test (unpublished results).

The above findings led us to develop and validate a new mucosal IgA assay, using saliva, nasal and OPF samples, as a confirmatory or screening test for detection of persistently FMDV infected cattle.

\section{Materials and Methods}

\subsection{Animals and Sample Collection}

2.1.1. Single Dose O Manisa Vaccination and O UKG Challenge Experiments

Saliva, nasal and OPF samples were collected from four vaccine challenge experiments, each consisting of 25 Holstein-Friesian cattle, aged 4-8 months, carried out in biosecurity containment at the Pirbright Institute, Pirbright, U.K. Experiments had been designed to evaluate vaccine induced protection against challenge 21 or 10 days later, by contact with animals infected with a semi-heterologous FMDV strain. Animals in each experiment were assigned with 2 letter identifiers (UV, UY, VD/VE, and VH) coupled with animal-specific numbers. In each experiment, 20 cattle were vaccinated with oil adjuvanted FMDV type O Manisa emergency vaccine obtained from the UK FMDV antigen reserve, and compliant with the Office International des Epizooties (OIE) guidelines for freedom from non-structural proteins (NSPs). In each experiment five cattle were included as unvaccinated control. In UV and VH animal experiments, the vaccine used represented a formulation previously determined to have a potency of $18 \mathrm{PD}_{50}$ (according to the European pharmacopoeia cattle potency test), whereas in the UY, VD/VE animal experiments, a 10 times increase per bovine dose of antigen was included in the vaccine formulation. The challenge was performed by 5 days of contact with donor cattle that had been infected with O UKG 34/2001 by tongue inoculation. The experimental details have been extensively reported in earlier publications $[14,20-23]$ and are shown in the Table S1.

\subsubsection{Repeat Dose O Manisa Vaccination and O UKG Challenge Experiment}

In order to evaluate the effect of multiple vaccinations on anti-FMDV mucosal antibody titres, mucosal fluids (saliva, nasal, probang) were collected from cattle vaccinated with $\mathrm{O}$ Manisa vaccine $\left(18 \mathrm{PD}_{50}\right)$ three times at 21-day intervals and then challenged with $\mathrm{O}$ UKG 34/2001 FMD virus on the 35th day after the 3rd vaccination. This challenge was 
performed by contact with the donor animals from the above-mentioned UY experiment starting six days after FMDV tongue inoculation.

\subsubsection{Naïve Cattle}

To determine the specificity and cut-off value of the mucosal IgA test, saliva fluid samples $(n=875)$, were collected from cattle that had had no contact with FMDV. These cattle comprised animals from the Mayfield dairy farm, Compton, UK; from the Republic of Ireland; from animals sourced from the UK, at the beginning of vaccine challenge/vaccine potency experiments at the Pirbright Institute, before any administration of vaccine or virus. Similarly, nasal fluids $(n=224)$ and OPF samples $(n=188)$ had been collected from individual naïve cattle before use in the different vaccine challenge/vaccine potency experiments and were used for the determination of cut-off values in nasal and probang IgA tests, respectively. Saliva samples were also collected from these animals for the cut-off estimation of the saliva test.

\subsubsection{Sample Collection}

Saliva and nasal fluids were collected from the vestibule and underneath the tongue of the mouth and from the nostril, respectively by using a 1/6th portion of regular size cotton tampons (Tampax ${ }^{\circledR}$, Hungary) or Sarsted saliva collection kits ${ }^{\circledR}$ pre-dampened by the addition of $0.5 \mathrm{~mL}$ of phosphate buffered saline ( $\mathrm{pH}$ 7.5). The tampon swab was held by forceps to collect the samples. In the laboratory, approximately $1-2 \mathrm{~mL}$ of saliva/nasal fluid samples were extracted from each tampon/saliva collection kit by compression within the barrel of a syringe or by centrifugation for $10 \mathrm{~min}$ at $1862 \times \mathrm{g}$ before storage at $-20^{\circ} \mathrm{C}$ [14] Samples from tampons and kits were compared for volume and presence of antibody and were found equivalent. OPF were collected by using a probang sampling cup [24]. Blood samples were also collected for detection of anti-NSP antibody in serum using Vacutainers ${ }^{\circledR}$ (BD, USA).

\subsection{Test Procedures}

\subsubsection{Virological Tests}

Results of virus isolation (VI) and RT-PCR from probang fluids that had already been documented [14,20-23], were considered as the gold standard for the detection of carrier cattle. Probang sample positive animals, either by RT-PCR or by VI or by both techniques after 28 days post-challenge, were considered as carrier animals. As shown in previous studies [1,3], perhaps due to variability in the material collected by probang sampling, intermittently positive virological results may be obtained in both the tests. However, the animals were considered as carriers if the results were positive on at least one occasion on or after 28 days post-challenge. The same approach was used to characterise the samples and animals from the experimental cattle vaccinated three times before challenge that have not been previously reported.

\subsubsection{Serological Tests}

Pre-documented results [14,20-23] of the PrioCHECK ${ }^{\circledR}$-NSP assay (Prionics AG) have been used to estimate the concordance between 3ABC-NSP test and IgA assay for the detection of carriers. ThePrioCHECK ${ }^{\circledR}$-NSP assay was also used to measure anti-NSP antibodies in samples from the cattle vaccinated three times prior to challenge. The PrioCHECK ${ }^{\circledR}-\mathrm{NSP}$ is a competitive blocking ELISA that measures the competition between serum anti-FMDV NSP antibody and a NSP-3B specific monoclonal antibody for binding to a recombinant $3 \mathrm{ABC}$ NSP of FMDV $[25,26]$. The assay was conducted as per the manufacturer's instructions. 


\subsubsection{Indirect Sandwich ELISA for Detection of IgA Antibodies}

An indirect ELISA for the detection of IgA antibodies to structural proteins has been developed previously [14]. However, to increase the specificity of the salivary IgA test, two separate negative antigens (FMDV SAT2 and BHK-21 cell lysate) and a blocking buffer were included as negative controls in the ELISA plate. The final OD was calculated after deducting the OD value of the negative control from the OD value of the specific antigen. The best negative control selected in the salivary IgA assay has been used subsequently in the nasal and OPF IgA ELISA.

Odd-numbered columns of the ELISA plates (Nunc, Roskilde, Denmark) were coated with $0.1 \mathrm{M}$ carbonate/bicarbonate buffer, $\mathrm{pH}$ 8.0-8.4 (50 $\mu \mathrm{L} /$ well) containing rabbit antiFMDV antiserum (O Manisa), while the even-numbered columns were coated with SAT2 rabbit anti-FMDV antiserum. In addition to the SAT2 control, in the case of the salivary IgA test, BHK-21 cell lysate and blocking buffer control wells were coated with rabbit anti-FMDV antiserum (O Manisa). After incubating the coated plate at $4{ }^{\circ} \mathrm{C}$ overnight, the respective pre-titrated antigens diluted in blocking buffer were added. Following washing, test samples ( $7 \mu \mathrm{L}$ of saliva/probang or $2 \mu \mathrm{L}$ of nasal fluid) along with in-house test positive and negative standards (controls) were added to the blocking buffer $(43 / 48 \mu \mathrm{L})$ in the plates and incubated for $1 \mathrm{~h}$ at $37^{\circ} \mathrm{C}$. After a further wash, specific bovine $\operatorname{IgA}$ was detected using a polyclonal rabbit anti-bovine IgA HRPO conjugate. Plates were finally washed three times and the test was developed by the addition of substrate. The reaction was stopped after $10 \mathrm{~min}$ by addition of $1 \mathrm{M}$ sulphuric acid and the optical density (OD) from plates were read on a multi-channel spectrophotometer (Molecular Devices, Inc., San Jose, CA, USA) at $490 \mathrm{~nm}\left(\mathrm{~A}_{490}\right)$.

The test results were expressed in terms of percentage of positivity (PP) which was determined as follows:

$$
\begin{gathered}
O D_{\text {corrected }}=O D_{\text {sample }}-O D_{\text {control }} \\
P P=\frac{100 \times O D_{\text {corrected(test })}}{O D_{\text {corrected(positive control) }}}
\end{gathered}
$$

\subsection{Statistical Analysis}

Comparison of the anti-FMDV IgA response between the carrier and non-carrier groups was performed using the student's t-test, whereas analysis of variance (ANOVA) was used to assess differences in the anti-FMDV IgA antibody level estimated from the nasal, saliva and oro-pharyngeal fluids. To determine the performance of the IgA-ELISA, nonparametric estimations of receiver operating characteristic (ROC) curves were run in Stata 14 SE (Stata Corp, LP), evaluating the diagnostic performance (sensitivity (Se) and specificity (Sp)) at different cut-off points. The maximum-likelihood ROC model was used to estimate the area under the curve (AUC) with associated $95 \%$ confidence intervals. A Bayesian model was further parametrised for assessing the sensitivity and specificity of the IgA-ELISA using a probabilistic constraint framework) [27]. The model was computed using WinBUGS 1.4.3 (MRC Biostatistics unit, Cambridge, UK) [28]. The MCMC chain was run for 50,000 with burn-in set at $10 \%$ of the chain.

\subsection{Ethics Statement}

All calf experiments were performed under the regulations of the Home Office Scientific Procedures Act (1986) of the United Kingdom, and had been approved by The Pirbright Institute Animal Welfare \& Ethical Review Body. 


\section{Results}

\subsection{Detection of FMDV Carrier Cattle by VI and RT-PCR}

Out of 80 vaccinated and 20 unvaccinated control cattle from four vaccine-challenge experiments involving a single dose of vaccine, 32 and 5 cattle were detected as FMDV carriers, respectively. The carrier status was identified by combining the VI and real-time RT-PCR tests (VI + RT-PCR). Analyses of OPF samples for detection of carriers in UV, UY, VD/VE and VH have been previously published [12,14,21]. All cattle that received multiple vaccine doses, were clinically protected against FMDV after challenge. Indeed, no virus/viral genome was identified by VI and RT-PCR from the OPF samples collected from the multiple vaccinated-challenged cattle.

\subsection{Development and Validation of IgA ELISA for the Evaluation of Anti-FMDV IgA Antibody} Response in the Mucosal Fluids

3.2.1. Selection of Suitable Negative Antigen Control to Increase the Sensitivity and Specificity of the Salivary IgA Assay

A test of equality of ROC areas was done using saliva samples of known uninfected and infected cattle tested using IgA assays constructed with the three different antigen controls (i.e., FMDV SAT2 and BHK-21 cell lysate, and blocking buffer). IgA ELISA data on saliva samples from naive uninfected cattle and from vaccinated uninfected cattle were used separately (Tables 1 and 2). ROC curves were plotted using the IgA-ELISA data originated from 32 cattle during the vaccination period (vaccinated uninfected cattle saliva, $n=78$ ) and then after they were infected and became persistently infected (vaccinated persistently infected cattle saliva samples, $n=300$ ), the samples from carrier cattle were collected at weekly intervals between 28 and 168 days post challenge (dpc). Although the AUC (0.93) estimated for both the BHK-21 and SAT2 negative antigen controls was relatively larger than that of the blocking buffer $(\mathrm{BB})$ control $(\mathrm{AUC}=0.909)$ (Figure 1), the sensitivity for the detection of FMDV carrier by IgA-ELISA was found to be highest using the SAT2 antigen control (Table 1). Therefore, considering the estimated sensitivity at different cut-off points (Table 1), the SAT2 antigen control was selected as the most suitable negative antigen control for the serotype O specific IgA ELISA.

Table 1. Diagnostic performance of IgA-ELISA estimated using saliva samples from vaccinated uninfected and vaccinated infected cattle. Diagnostic parameters are reported at different cut-off points using the three different negative antigen controls used to construct the IgA-ELISAs. PP = percentage of positivity; $\mathrm{LR}+=$ likelihood ratio for a positive test; $\mathrm{LR}-=$ likelihood ratio for a negative test.

\begin{tabular}{cccccc}
\hline Negative Ag Control & PP & Sensitivity (Se) & Specificity (Sp) & LR+ & LR- \\
\hline & 20 & $84.46 \%$ & $94.87 \%$ & 16.470 & 0.164 \\
SAT 2 & 25 & $78.38 \%$ & $84.87 \%$ & 15.284 & 0.228 \\
& 30 & $73.65 \%$ & $96.15 \%$ & 19.149 & 0.274 \\
& 35 & $69.26 \%$ & $97.44 \%$ & 27.010 & 0.315 \\
& 40 & $65.54 \%$ & $97.44 \%$ & 25.561 & 0.354 \\
\hline \multirow{3}{*}{ BHK-21 } & 20 & $77.10 \%$ & $96.15 \%$ & 20.047 & 0.238 \\
& 25 & $71.72 \%$ & $98.72 \%$ & 55.939 & 0.286 \\
& 30 & $66.33 \%$ & $98.72 \%$ & 51.727 & 0.341 \\
& 35 & $61.28 \%$ & $98.72 \%$ & 47.798 & 0.392 \\
& 40 & $57.91 \%$ & $98.72 \%$ & 45.172 & 0.426 \\
\hline \multirow{3}{*}{ Blocking-Buffer } & 20 & $73.40 \%$ & $96.15 \%$ & 19.084 & 0.277 \\
& 25 & $67.78 \%$ & $97.44 \%$ & 26.394 & 0.332 \\
& 30 & $62.96 \%$ & $97.44 \%$ & 24.556 & 0.380 \\
& 35 & $60.27 \%$ & $97.44 \%$ & 23.505 & 0.408 \\
& 40 & $54.55 \%$ & $97.44 \%$ & 21.273 & 0.466 \\
\hline
\end{tabular}


Table 2. Diagnostic performance of IgA-ELISA estimated using saliva samples from naïve uninfected and vaccinated infected cattle. Diagnostic parameters are reported at different cut-off points using the three different negative antigen controls used to construct the IgA-ELISAs. PP = percentage of positivity; $\mathrm{LR}+=$ likelihood ratio for a positive test; $\mathrm{LR}-=$ likelihood ratio for a negative test.

\begin{tabular}{cccccc}
\hline Negative Ag Control & PP & Sensitivity (Se) & Specificity (Sp) & LR+ & LR- \\
\hline & 20 & $84.12 \%$ & $91.77 \%$ & 10.039 & 0.173 \\
SAT 2 & 25 & $78.04 \%$ & $94.82 \%$ & 15.060 & 0.232 \\
& 30 & $73.65 \%$ & $96.34 \%$ & 19.647 & 0.274 \\
& 35 & $68.92 \%$ & $97.60 \%$ & 27.178 & 0.319 \\
& 40 & $65.54 \%$ & $98.74 \%$ & 49.538 & 0.349 \\
\hline \multirow{3}{*}{ BHK-21 } & 20 & $77.10 \%$ & $96.69 \%$ & 23.311 & 0.237 \\
& 25 & $71.72 \%$ & $97.02 \%$ & 24.092 & 0.291 \\
& 30 & $66.33 \%$ & $97.35 \%$ & 25.067 & 0.346 \\
& 35 & $61.28 \%$ & $97.79 \%$ & 27.790 & 0.396 \\
& 40 & $57.91 \%$ & $98.24 \%$ & 32.829 & 0.428 \\
\hline \multirow{3}{*}{ Blocking-Buffer } & 20 & $73.40 \%$ & $88.42 \%$ & 6.340 & 0.301 \\
& 25 & $67.68 \%$ & $92.06 \%$ & 8.525 & 0.351 \\
& 30 & $62.96 \%$ & $94.05 \%$ & 10.575 & 0.394 \\
& 35 & $59.60 \%$ & $95.59 \%$ & 13.513 & 0.423 \\
& 40 & $54.55 \%$ & $96.36 \%$ & 14.992 & 0.472 \\
\hline
\end{tabular}

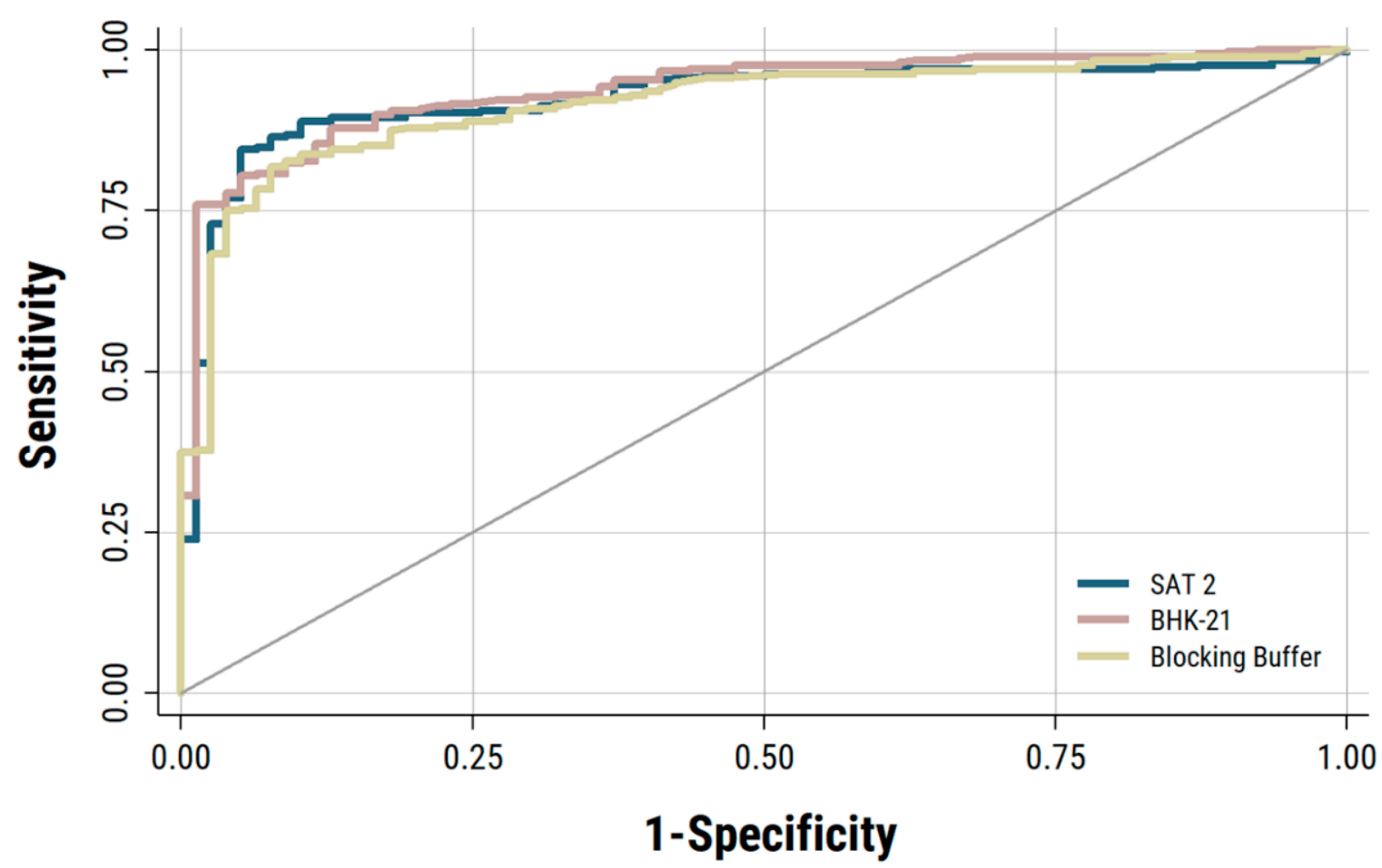

Figure 1. ROC curves estimated for IgA-ELISA using saliva samples collected from vaccinated un-infected and vaccinatedinfected cattle. Three different negative antigen controls (FMDV SAT2, BHK-21 cell lysate, and a blocking buffer) were used to construct the IgA ELISA.

Similarly, ROC curves were also plotted using the IgA-ELISA data from saliva samples collected from 875 individual naïve cattle and from saliva samples $(n=300)$ collected from 32 vaccinated persistently infected cattle collected at weekly intervals between 28 and $168 \mathrm{dpc}$ (Figure 2). The AUC for the BHK-21 antigen control was found to be the highest among the three antigen controls (Figure 2), but the sensitivity for the detection of FMDV carrier animals by IgA-ELISA was again found to be highest using the SAT-2 antigen 
control (Table 2). Therefore, the SAT2 control was selected as the suitable negative antigen control for subsequent analysis and validation of IgA-ELISA using saliva, nasal and OPF.

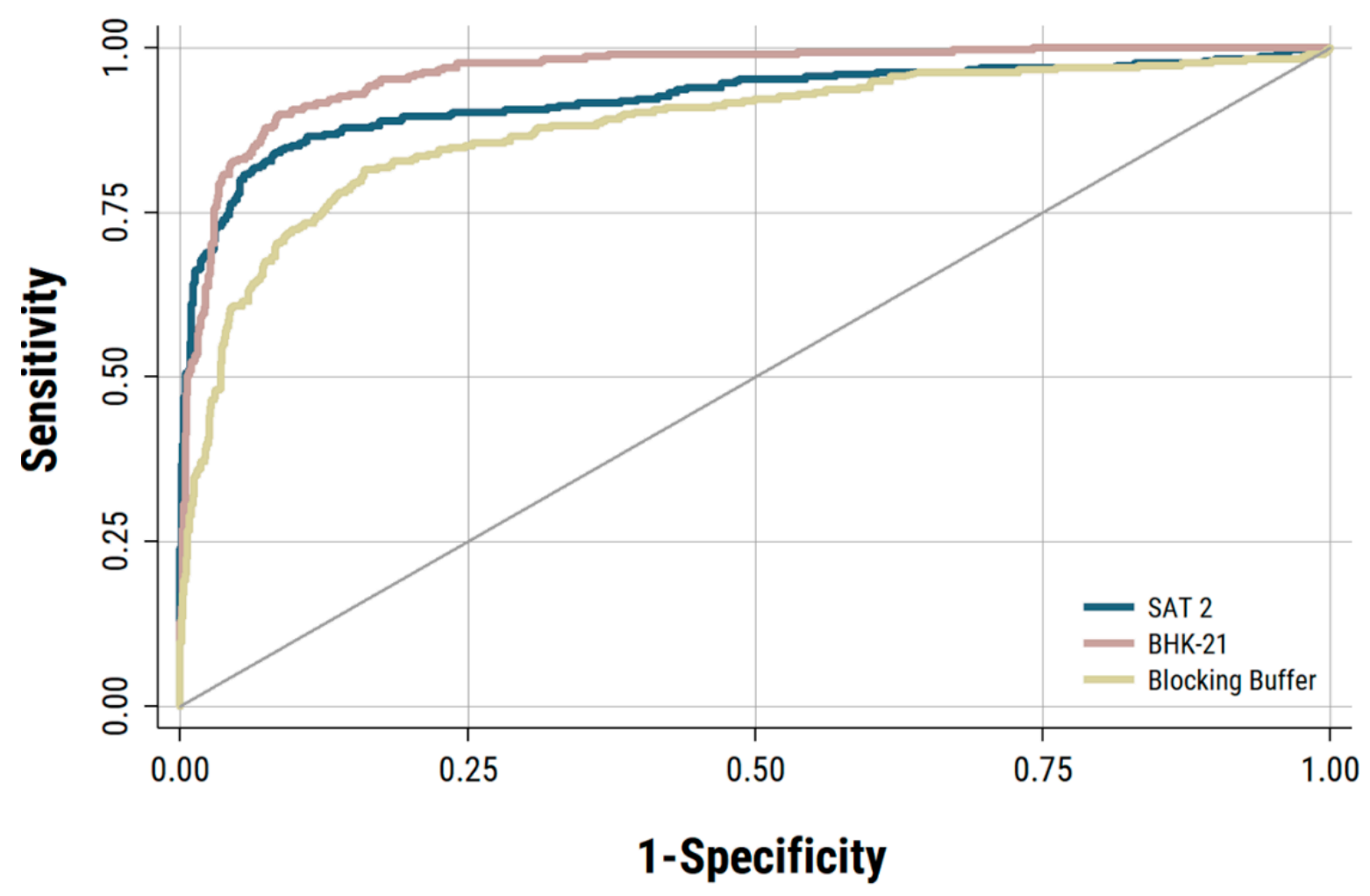

Figure 2. ROC curves estimated for IgA-ELISA using saliva samples collected from naïve un-infected and vaccinatedinfected cattle. Three different negative antigen controls (FMDV SAT2, BHK-21 cell lysate, and a blocking buffer) were used to construct the IgA ELISA.

3.2.2. Comparison of Diagnostic Sensitivity and Diagnostic Specificity for Anti-FMDV IgA-ELISAs Using Saliva, Nasal and Oro-Pharyngeal Fluid Samples

Diagnostic characteristics of the salivary IgA ELISA at different cut-off points were estimated by the non-parametric ROC analysis method (Tables 1 and 2). Considering the Se (sensitivity) and Sp (specificity), a suitable cut-off value of 35 percentage of positivity (PP) was selected for the salivary IgA-ELISA. At 35 PP a Se of $68.92 \%$ and Sp of $97.60 \%$ was obtained for the salivary IgA ELISA (Table 2). For the determination of the diagnostic parameters of the nasal IgA-ELISA, nasal fluids from individual naïve cattle $(n=224)$ and carrier cattle (300 samples from 32 carrier animals) collected at weekly intervals between 28 and $168 \mathrm{dpc}$ were used. For the nasal IgA-ELISA, a cut-off value of $35 \mathrm{PP}$ was selected with a Se of $76.53 \%$ and a Sp of $99.11 \%$ (Table 3). Similarly, 188 OPF samples from individual naïve cattle and $276 \mathrm{OPF}$ samples from carrier cattle $(n=37)$ collected at weekly intervals between 28 and $161 \mathrm{dpc}$, were analysed by the IgA-ELISA assay and a suitable cut-off value of 35 PP was reported (Table 4). At the 35 PP, a Se of 59.35\% and a Sp of $99.47 \%$ was obtained (Table 4). 
Table 3. Diagnostic performance of the nasal IgA-ELISA. Diagnostic parameters are reported at different cut-off points. $\mathrm{PP}=$ percentage of positivity; $\mathrm{LR}+=$ likelihood ratio for a positive test; LR $-=$ likelihood ratio for a negative test. Diagnostic cut-off is set at 35PP.

\begin{tabular}{ccccc}
\hline PP & Se & Sp & LR+ & LR - \\
\hline 10 & $90.48 \%$ & $94.20 \%$ & 15.589 & 0.101 \\
20 & $84.35 \%$ & $96.88 \%$ & 26.993 & 0.161 \\
30 & $78.23 \%$ & $97.77 \%$ & 35.048 & 0.223 \\
35 & $76.53 \%$ & $99.11 \%$ & 85.714 & 0.237 \\
40 & $74.49 \%$ & $99.11 \%$ & 83.428 & 0.255 \\
45 & $69.73 \%$ & $100 \%$ & - & 0.303 \\
50 & $65.65 \%$ & $100 \%$ & - & 0.343 \\
\hline
\end{tabular}

Table 4. Diagnostic performance of the probang IgA-ELISA. Diagnostic parameters are reported at different cut-off points. $\mathrm{PP}=$ percentage of positivity; $\mathrm{LR}+=$ likelihood ratio for a positive test; $\mathrm{LR}-$ $=$ likelihood ratio for a negative test. Diagnostic cut-off is set at 35PP.

\begin{tabular}{ccccc}
\hline PP & Se & Sp & LR+ & LR - \\
\hline 10 & $87.05 \%$ & $93.65 \%$ & 13.710 & 0.138 \\
20 & $73.38 \%$ & $97.88 \%$ & 34.673 & 0.272 \\
30 & $64.39 \%$ & $98.94 \%$ & 60.847 & 0.360 \\
35 & $59.35 \%$ & $99.47 \%$ & 112.177 & 0.409 \\
40 & $56.47 \%$ & $99.47 \%$ & 106.738 & 0.438 \\
45 & $47.84 \%$ & $99.47 \%$ & 90.421 & 0.524 \\
50 & $38.13 \%$ & $99.47 \%$ & 72.065 & 0.622 \\
\hline
\end{tabular}

Since nasal sample-based IgA-ELISA performed better as compared to the saliva and OPF samples, the diagnostic Sp and Se of the nasal IgA-ELISA was also calculated using a Bayesian framework with probabilistic constraints. The Bayesian model provides a framework with multiple iterations to assess uncertainty in order to improve the accuracy of the ROC calculations. By using the Bayesian model, a specificity of $99.3 \%$ and a sensitivity of $85.4 \%$ was obtained for the nasal IgA-ELISA (Table 5).

Table 5. Diagnostic parameters estimated for each of the IgA-ELISA by Bayesian modelling using probabilistic constraints. Sensitivity and specificity estimates are expressed as median (95\% Bayesian Credible Interval).

\begin{tabular}{ccc}
\hline & Sensitivity & Specificity \\
\hline Saliva & $0.79(0.76-0.85)$ & $0.99(0.99-1.00)$ \\
Nasal & $0.85(0.80-0.93)$ & $0.99(0.99-1.00)$ \\
Probang & $0.70(0.64-0.78)$ & $0.99(0.99-1.00)$ \\
\hline
\end{tabular}

3.2.3. Comparative Anti-FMDV IgA Response in the Mucosal Fluid (Saliva, Nasal and Probang) Samples of Carrier and Non-Carrier Cattle

In all the mucosal fluids, the mean anti-FMDV IgA titre remained below the cut-off value ( $35 \mathrm{PP}$ ) during the entire vaccination period (Figure 3). However, in the vaccinated carrier cattle but not in non-carriers, the mean IgA response in nasal fluid started to rise within 7-14 days post-challenge (dpc). In comparison, the IgA response both in saliva and probang samples was delayed up to 14-21 dpc (Figure 3). By $21 \mathrm{dpc}$, the nasal IgA response remained above the cut-off value whereas both the saliva and OPF IgA responses were seen to be positive only after $28 \mathrm{dpc}$. The mean anti-FMDV IgA response in mucosal fluids of all the 5 unvaccinated carrier cattle was found to be above the cut-off value only after 35 dpc (Figure 4). 


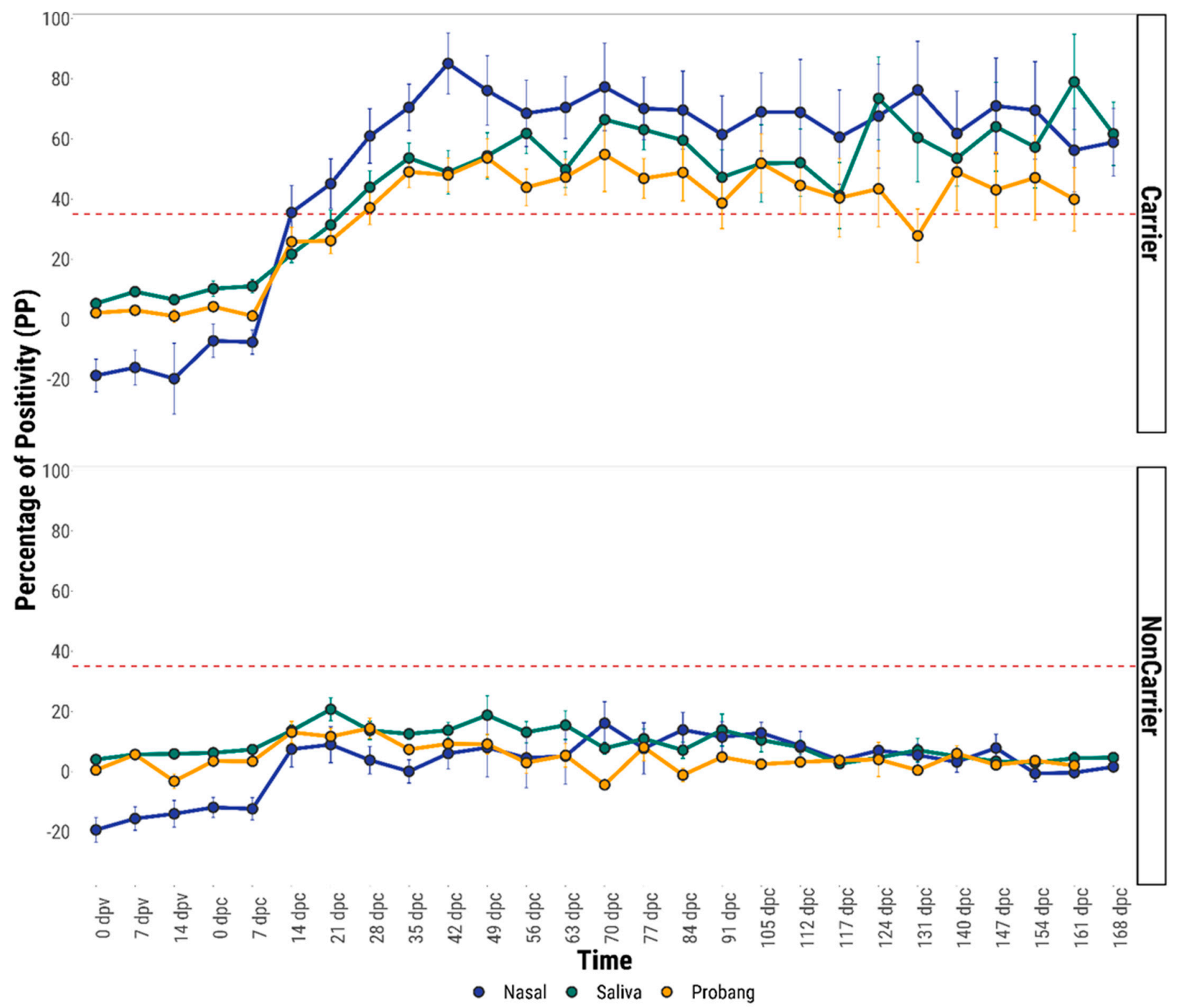

Figure 3. Comparative mean anti-FMDV IgA antibody responses in the mucosal (nasal, saliva and probang) fluids of vaccinated carrier and non-carrier cattle. The error bar represents the standard error. Red horizontal dashed lines indicate the diagnostic cut-off (35 PP).

The mean anti-FMDV IgA responses in all the mucosal fluids of vaccinated and unvaccinated carrier animals were seen to be significantly higher $(p \leq 0.05)$ than that of the respective non-carrier animals (Figures 3 and 4). In vaccinated carriers, a higher mean anti-FMDV IgA antibody response was observed in the nasal samples than the saliva and probang samples during many of the sampling days beyond 28 days post-challenge (dpc) (Figure 3). However, a statistically significant difference $(p \leq 0.05)$ in the anti-FMDV IgA response was found between the three fluids only on $35 \mathrm{dpc}(p=0.03), 42 \mathrm{dpc}(p=0.001)$ and $63 \mathrm{dpc}(p=0.04)$. 


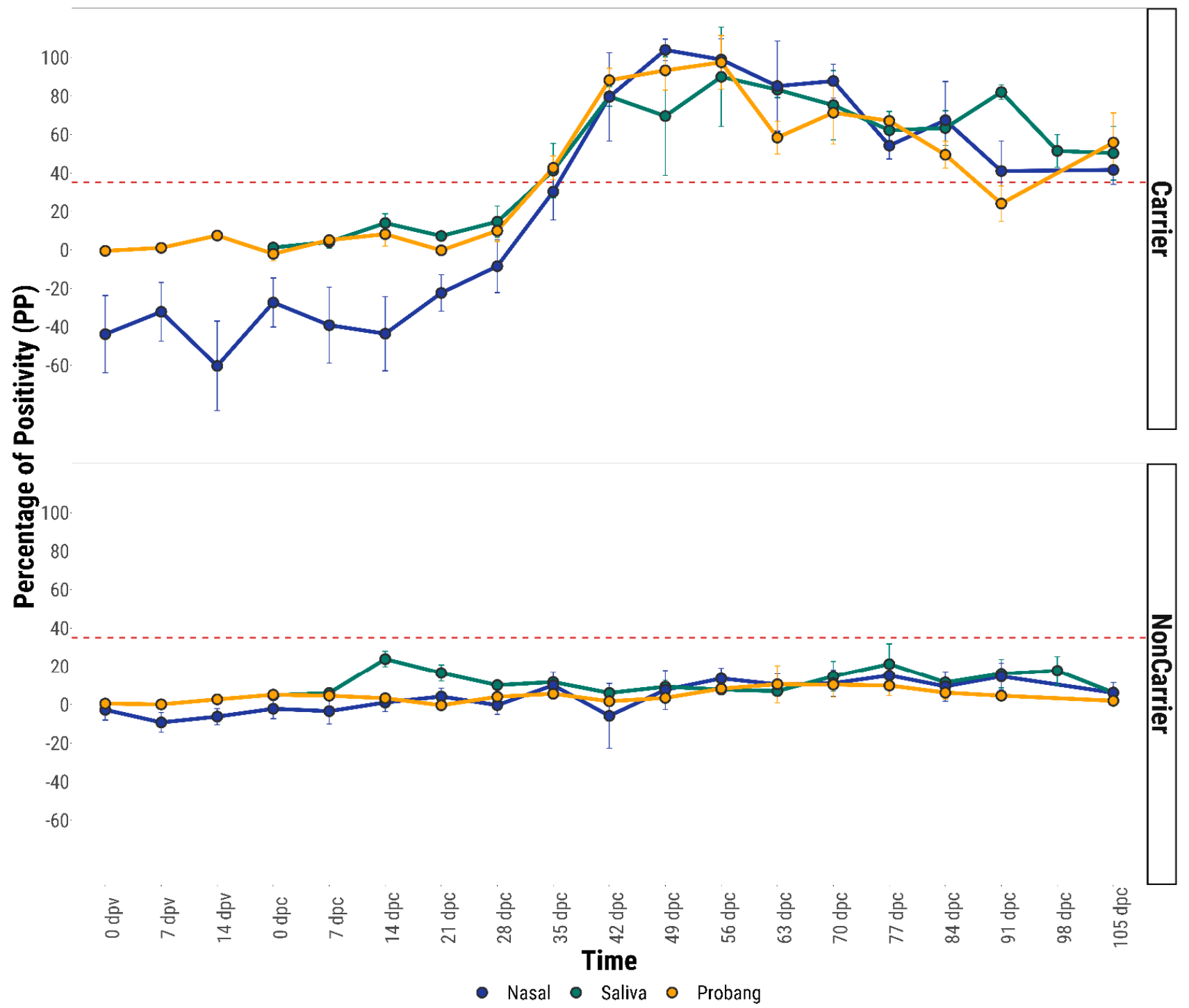

Figure 4. Comparative mean anti-FMDV IgA response in the mucosal (nasal, saliva and probang) fluids of unvaccinated carrier and non-carrier animals. The error bars represent the standard error. Red horizontal dashed lines indicate the diagnostic cut-off (35 PP).

3.2.4. Analysis of Sensitivity Concordance between Nasal IgA-ELISA and PrioCHECK-NSP Test for the Detection of FMDV Carrier Cattle

As the FMDV NSP antibody assay has been used for the detection of FMDV carrier cattle, the level of concordance between the commercially validated PrioCHECK-NSP test and the nasal IgA-ELISA for the detection of FMDV carriers was determined. Out of 32 vaccinated-challenged carrier cattle detected by VI and/or RT-PCR, a total of 29 cattle were scored positive either by the nasal IgA assay or by the PrioCHECK-NSP test (Table 6). However, from these 29 detected carrier cattle, only 27 cattle were found to be concordantly positive by both IgA and NSP antibody tests. The PrioCHECK-NSP assay scored another 18 vaccinated cattle as seropositive after challenge, in addition to the 29 cattle that were virologically confirmed carriers (Table 6). Combining the results from the nasal IgA and the NSP antibody test, 31 out of 32 vaccinated carriers were scored positive by one or both tests, resulting in an enhanced sensitivity of $96.87 \%$. 
Table 6. Validation and comparison of nasal IgA-ELISA test efficiency with NSP assay (PrioCHECK-NSP) for detection of FMDV carriers. Assuming all carrier animals were detected by VI + RT-PCR assay $(100 \%)$, all the other percentage values were calculated. $\mathrm{VI}=$ virus isolation; NSP = non-structural protein.

\begin{tabular}{|c|c|c|c|c|c|c|c|}
\hline$\underset{\text { Experiments }}{\text { Animal }}$ & $\begin{array}{c}\text { Clinically In- } \\
\text { fected/Vaccinated } \\
\text { Challenged } \\
\text { Animals }\end{array}$ & $\begin{array}{c}\text { Vaccinated } \\
\text { Carriers } \\
\text { Detected by } \\
\text { VI + RT-PCR }\end{array}$ & $\begin{array}{c}\text { Carriers } \\
\text { Detected by } \\
\text { PrioCHECK- } \\
\text { NSP } \\
\text { Test }\end{array}$ & $\begin{array}{l}\text { NSP Serocon- } \\
\text { version by } \\
\text { PrioCHECK- } \\
\text { NSP } \\
\text { Assay. }\end{array}$ & $\begin{array}{c}\text { Carriers } \\
\text { Detected by } \\
\text { Nasal } \\
\text { IgA-ELISA }\end{array}$ & $\begin{array}{c}\text { Carriers } \\
\text { Concordantly } \\
\text { Detected by } \\
\text { both } \\
\text { PrioCHECK- } \\
\text { NSP and IgA } \\
\text { Test }\end{array}$ & $\begin{array}{c}\text { Carriers } \\
\text { Detected by } \\
\text { Either } \\
\text { PrioCHECK- } \\
\text { NSP or IgA } \\
\text { Test or Both }\end{array}$ \\
\hline UV & $0 / 20$ & 9 & 7 & 10 & 8 & 7 & 9 \\
\hline UY & $0 / 20$ & 3 & 3 & 7 & 3 & 3 & 3 \\
\hline $\mathrm{VH}$ & $5 / 20$ & 9 & 9 & 18 & 9 & 9 & 9 \\
\hline $\mathrm{VD} / \mathrm{VE}$ & $6 / 20$ & 11 & 10 & 12 & 9 & 8 & 10 \\
\hline TOT & $11 / 80$ & $32(100 \%)$ & $29(90.62 \%)$ & 47 (146.87\%) & $29(90.62 \%)$ & 27 (84.37\%) & 31 (96.87\%) \\
\hline
\end{tabular}

Multiple samples were available from many of the vaccinated and challenged cattle and, in some cases, the results changed over time so that both virological and serological tests sometimes failed to detect carriers at one or more timepoints. Therefore, it was decided to calculate the sensitivity concordance by using the data originating from individual sampling days on and after 28 days post challenge. Considering the number of known carrier cattle on different days of sampling period as $100 \%$, the sensitivity of all three assays were calculated (Table 7). The sensitivity of the combined VI + RT-PCR assay for the detection of carriers varied from 0 to $78 \%$ (Table 6). The sensitivity of IgA-ELISA for detection of FMDV persistent cattle varied from 62 to $88 \%$, while the performance of PrioCHECK-NSP test for the detection of carriers varied from 75 to $90 \%$ (Table 7). By combining both the IgA assay and NSP test, 75-100\% of known carriers were detected by one or both tests (Table 7).

Table 7. Comparative sensitivities of various virological and serological assays for detection of vaccinated and subsequently infected carrier cattle. Considering number of known carriers on different days of sampling period as $100 \%$, sensitivities for all the other assays were calculated. Dpc = days post-challenge; VI = virus isolation; NSP = non-structural protein.

\begin{tabular}{cccccc}
\hline $\begin{array}{c}\text { Sampling } \\
\text { Days (dpc) }\end{array}$ & $\begin{array}{c}\text { No. of } \\
\text { Carriers }\end{array}$ & $\begin{array}{c}\text { Carriers Detected } \\
\text { by VI + RT-PCR }\end{array}$ & $\begin{array}{c}\text { Carriers Detected } \\
\text { by Nasal IgA }\end{array}$ & $\begin{array}{c}\text { Carriers Detected by } \\
\text { PrioCHECK-NSP }\end{array}$ & $\begin{array}{c}\text { Carriers Detected by } \\
\text { IgA+ PrioCHECK }\end{array}$ \\
\hline 28 & $32(100 \%)$ & $25(78.1 \%)$ & $24(75 \%)$ & $27(84.37 \%)$ & $30(93.75 \%)$ \\
35 & $32(100 \%)$ & $25(78.1 \%)$ & $25(78 \%)$ & $28(87.5 \%)$ & $30(93.75 \%)$ \\
42 & $23(100 \%)$ & $12(52.17 \%)$ & $18(78.26 \%)$ & $18(78.26 \%)$ & $20(86.95 \%)$ \\
49 & $21(100 \%)$ & $14(66.66 \%)$ & $17(80.95 \%)$ & $16(76.19 \%)$ & $20(95.23 \%)$ \\
55 & $21(100 \%)$ & $13(61.90 \%)$ & $16(76.19 \%)$ & $18(85.71 \%)$ & $19(90.47 \%)$ \\
63 & $21(100 \%)$ & $7(33.33 \%)$ & $17(80.95 \%)$ & $18(85.71 \%)$ & $19(90.47 \%)$ \\
77 & $21(100 \%)$ & $10(47.61 \%)$ & $16(76.19 \%)$ & $19(90.47 \%)$ & $19(90.47 \%)$ \\
84 & $12(100 \%)$ & $4(33.33 \%)$ & $10(83.33 \%)$ & $10(83.34 \%)$ & $12(100 \%)$ \\
91 & $12(100 \%)$ & $3(25 \%)$ & $8(66.67 \%)$ & $10(83.34 \%)$ & $11(91.66 \%)$ \\
105 & $12(100 \%)$ & $2(16.66 \%)$ & $9(75 \%)$ & $9(75 \%)$ & $10(83.34 \%)$ \\
112 & $9(100 \%)$ & $1(11.11 \%)$ & $7(77.76 \%)$ & $7(77.76 \%)$ & $7(77.76 \%)$ \\
117 & $9(100 \%)$ & $1(11.11 \%)$ & $5(55.56 \%)$ & $7(77.76 \%)$ & $7(77.76 \%)$ \\
124 & $9(100 \%)$ & $2(22.22 \%)$ & $6(66.67 \%)$ & $7(77.76 \%)$ & $8(88.89 \%)$ \\
131 & $8(100 \%)$ & $1(12.5 \%)$ & $6(75 \%)$ & $6(75 \%)$ & $6(75 \%)$ \\
140 & $8(100 \%)$ & $0(0 \%)$ & $6(75 \%)$ & $6(75 \%)$ & $6(75 \%)$ \\
147 & $8(100 \%)$ & $2(25 \%)$ & $5(62.5 \%)$ & $6(75 \%)$ & $6(75 \%)$ \\
154 & $8(100 \%)$ & $1(12.5 \%)$ & $6(75 \%)$ & $6(75 \%)$ & $6(75 \%)$ \\
161 & $8(100 \%)$ & $2(25 \%)$ & $5(62.5 \%)$ & $6(75 \%)$ & $6(75 \%)$ \\
168 & $8(100 \%)$ & $1(12.5 \%)$ & $7(87.5 \%)$ & $6(75 \%)$ & $7(87.5 \%)$ \\
\hline
\end{tabular}


3.2.5. Anti-FMDV IgA and Anti-NSP Antibody Responses in Cattle Challenged after Repeated Vaccination

Repeatedly vaccinated cattle $(n=6)$ were clinically protected and none of them scored as carriers after $28 \mathrm{dpc}$ by VI or RT-PCR tests (data not shown). One animal (VC14) scored positive for anti-FMDV NSP antibody by PrioCHECK-NSP assay after the third vaccination ( $56 \mathrm{dpv}$ ), while animal VC19 scored positive, just above the threshold cut-off, on $63 \mathrm{dpv}$ and $70 \mathrm{dpv}$. However, these two cattle became seropositive after challenge (Figure 5). FMDV specific IgA antibody responses in the nasal samples of the cattle remained below the threshold ( $35 \mathrm{PP}$ ) value at all times prior to challenge (Figure 5). However, a rise in IgA antibody was found in VC15 immediately after the second vaccination (Figure 5), albeit remaining below the threshold value. After two to three weeks of challenge, only one animal (VC 14) scored positive in the IgA antibody ELISA.
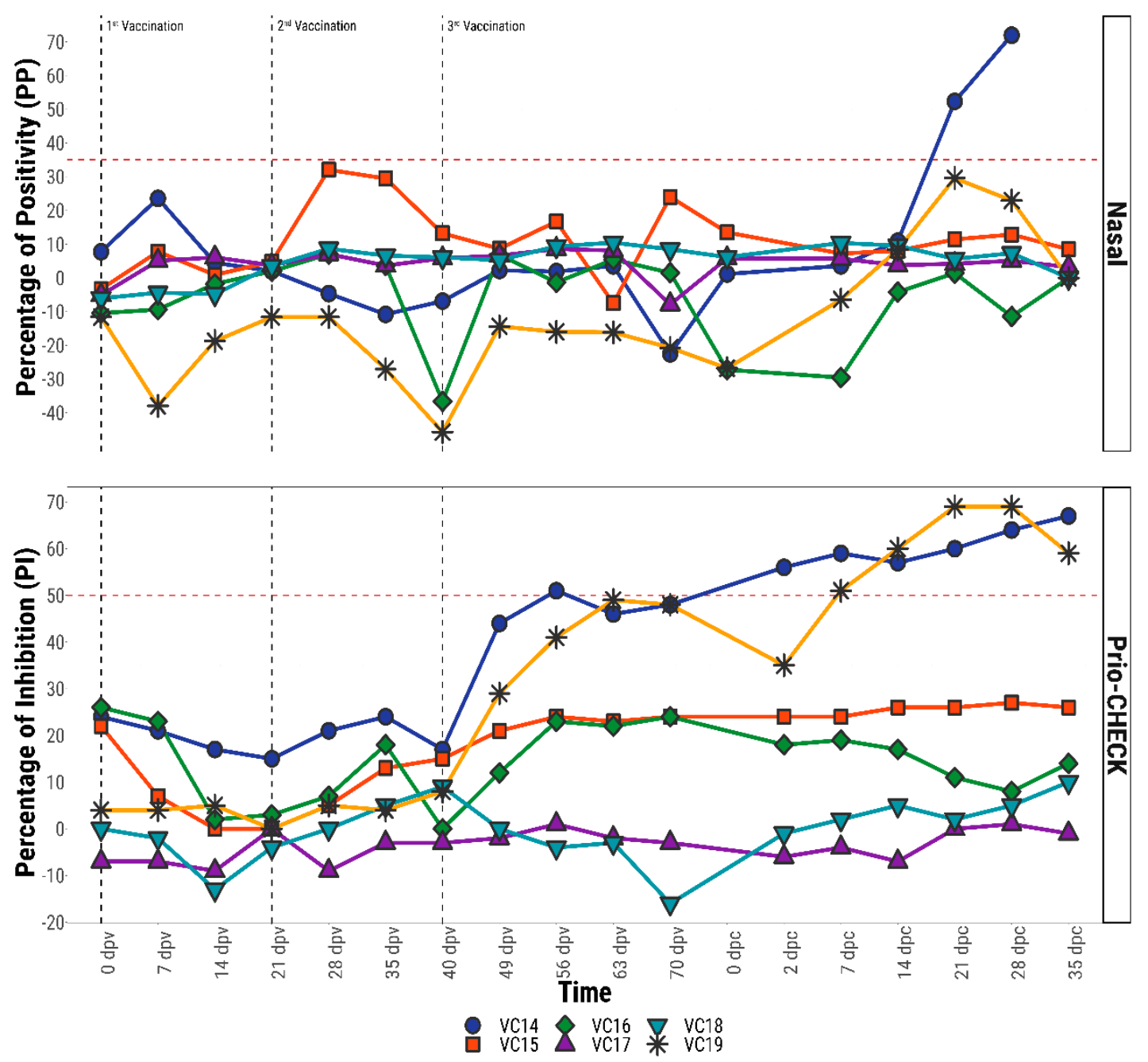

Figure 5. Antibody responses (FMDV specific IgA in nasal fluid and anti-NSP antibody in serum) after repeated vaccination in cattle. Vertical dashed black lines indicate the day of vaccination, whilst the horizontal dashed red lines indicate the diagnostic cut-offs for both the Nasal IgA-ELISA (35 PP) and the Prio-CHECK NSP ELISA (50 PI) tests. IgA antibody titre expressed as percentage of positivity (PP) values and anti-NSP antibody titre expressed as percentage of inhibition (PI) values.

\section{Discussion}

Since FMDV carriers may be considered to pose a risk of passing on infection, to regain the FMD-free status without ongoing vaccination for the purpose of international trade, with or without the use of temporary emergency vaccination, post-outbreak surveillance is 
required to demonstrate the absence of infection [29]. Virus isolation and RT-PCR are two established methods to detect virus or viral genome from oro-pharyngeal fluids collected using a metallic probang cup [30]. Virus isolation is as sensitive as RT-PCR during the early phase of infection, whereas RT-PCR detects more carriers in the later phase of infection in both cattle and sheep $[12,16]$. Even after combining both virus isolation and RT-PCR test results, at all the time points of sampling $100 \%$ carriers are not detected from the OPF samples collected sequentially in time [12], which is a major constraint in FMD surveillance. Besides this, the approach is cumbersome for mass screening after widespread use of emergency vaccines. Therefore, there is a need for alternative or supplementary methods to detect subclinical infection with FMDV in vaccinated herds.

The presence of anti-FMDV IgA antibody in saliva has been described as an indicator of oro-pharyngeal replication of FMDV [14]. Though an indirect IgA-ELISA using saliva samples to detect FMDV carrier cattle following vaccination and challenge exposure has been developed earlier, the assay was unsatisfactory on its diagnostic parameters. Therefore, an attempt has been made to develop and validate a modified IgA-ELISA targeting an increased specificity. Non-specificity in the assay may be either due to the high content of detached cells, proteases and tissue particles in the saliva samples [17], or due to the use of tissue culture derived inactivated crude antigen. In order to achieve a better diagnostic specificity, different negative antigen controls (heterologous SAT2 antigen, BHK-21 cell lysate and blocking buffer without any antigen) were evaluated in the newly developed salivary IgA-ELISA. Accordingly, the best format of the IgA-ELISA was selected with respect to both specificity and sensitivity using receiver-operating characteristic (ROC) curve analysis [31].

Normally, naïve samples are used to estimate the specificity of the diagnostic assay. However, considering the fact that serosurveillence should normally be conducted after emergency vaccination, it was decided to include saliva samples from both naive and vaccinated uninfected animals as a negative population control in ROC analysis for the selection of suitable negative antigen control.

The anti-FMDV IgA response was found to be higher in nasal fluids of FMDV vaccinated and sub-clinically infected carrier animals than saliva and probang fluids. However, both in the acute (for unvaccinated control animals) and in the persistent infection phase (both vaccinated and unvaccinated), the IgA levels were seen to be increased in all three mucosal fluids (saliva, nasal and probang) compared to samples from the vaccinated uninfected, vaccinated recovered and unvaccinated recovered animals. After 28 days postchallenge, the anti-FMDV IgA responses in the nasal and saliva fluids of both vaccinated and unvaccinated carrier animals were found to be significantly higher than those from the non-carriers. The high level of anti-FMDV IgA in the mucosal fluids may be due to the constant stimulation of the local mucosal immune system by persistent FMDV on the dorsal soft palate or naso-pharynx. Furthermore, the presence of IgA antibody on the nasal mucosal surface is a genuine FMD specific antibody response sustained by resident antibody secreting B-cells, rather than plasma transudation [17].

From the ROC estimations of diagnostic performance, a cut-off value of 35 percentage of positivity (35 PP) was determined for IgA-ELISA using the saliva, nasal and OPF. It was found that nasal IgA-ELISA performed better compared to saliva and OPF IgA-ELISA. With the cut-off value of $35 \mathrm{PP}$ a specificity of $99.11 \%$ and sensitivity of $76.53 \%$ was obtained for nasal IgA-ELISA by ROC analysis, whilst these parameters were found to increase after Bayesian model fitting $(\mathrm{Sp}=99.3 \%$ and $\mathrm{Se}=85.4 \%)$. As this test has similar sensitivity and specificity to the PrioCHECK-NSP test, the IgA assay can be used as screening test for the detection of carrier cattle. The international workshop on serological testing for FMD previously organised in Brescia, Italy, recommended the use of more than one NSP test to increase the overall diagnostic specificity [13]. In this case, the second confirmatory test should be selected with at least equal specificity and a good sensitivity. As the IgA test detects mucosal antibody and the PrioCHECK-NSP test detects anti-FMDV humoral antibody, using one of the tests for screening and the other as confirmatory test may help 
to increase the specificity and sensitivity to detect the FMDV carriers. The third option is to run both tests in parallel. In the present study, the concordance of sensitivity between the PrioCHECK-NSP and nasal IgA test was found to be $84.37 \%$. By combining both the nasal-IgA test and PrioCHECK-NSP test in parallel the overall test sensitivity was increased to $96.87 \%$.

Considering that there are no tests with perfect sensitivity and specificity, no serosurveillance can provide an absolute guarantee of freedom from infection. This applies even to a situation when the whole population has to be tested, e.g., the vaccinated cattle population in an EU country after emergency vaccination. Therefore, serosurveillance should be seen as part of a package of risk mitigation measures that will include movement restrictions, epidemiological tracing and clinical surveillance [13]. The problem of imperfect test sensitivity can be mitigated to some extent by attributing a status to a herd or population and then applying appropriate disease control measures. A herd could be classified as infected after one animal has reacted positively. However, as no test has a specificity of $100 \%$, many large herds may have to be considered infected due to false positive test results. As this is unacceptable, simultaneous testing by using serological methods with a very high specificity would be needed [13], albeit this would have the detrimental effect of decreasing the sensitivity. An alternative approach would be the culling of any animal reacting positive without classifying the herd as infected in the absence of further evidence of infection, e.g., clustering or increasing numbers of reactors or epidemiological information. In this case, the requirements for specificity could be lower and a higher sensitivity may be achievable [13].

During this study, the PrioCHECK NSP assay also scored positive for 18 cattle that were confirmed as non-carrier by VI and RT-PCR testing done at weekly intervals. This result confirms that NSP tests are not specific for the detection of carriers, as NSP antibody responses occur in infected animals that go on to eliminate FMDV. In addition, the NSP test may not be useful for individual animal screening, as cattle that have been vaccinated with high potency vaccine may produce stronger and earlier neutralising antibodies which limit the viral replication and sometimes fail to develop antibodies to NSPs following infection, but these animal could potentially become carriers [30,32]. Further in endemic countries where vaccine is not purified from NSP and multiple vaccination is practiced, there is an increased chance of NSP seroconversion without infection.

Although inactivated FMD vaccine, when administered parenterally, stimulates very little or no FMDV specific mucosal immune response [14,33], an immediate question arises about the effect of repeated vaccination on the mucosal anti-FMDV IgA response, particularly in the endemic countries where bi-annual prophylactic vaccination is carried out. In order to address this question, the opportunity was taken to test nasal and saliva samples for the detection of anti-FMDV specific antibody in repeatedly vaccinated animals (three times emergency vaccination at 21-day intervals). Anti-FMDV IgA antibody responses in nasal and saliva samples of six cattle remained below the cut-off value after multiple vaccinations, although a sub-threshold peak in IgA response was found for one animal, VC15, after the second vaccination. The anti-FMDV IgA responses in these repeatedly vaccinated animals suggest that the IgA assay may not be subject to non-specificity from multiple vaccinations even in endemic countries, although more animals need to be tested to confirm this. Furthermore, the analysis of serum samples originating from repeatedly vaccinated animals showed an increased humoral immune response of the anti-FMDV NSP antibody following the third vaccination in animals VC14 and VC19. This result supported the earlier finding [34] where the authors suggested that if animals received multiple doses of FMD vaccine, a small proportion of them might test positive without actual exposure to wild type FMD virus. The detection of NSP antibody in repeatedly vaccinated animals prior to challenge may be due to the presence of trace amounts of contaminating NSP proteins in the commercial FMD vaccine.

In conclusion, from the analysis of anti-FMDV IgA response in mucosal fluids, it is evident that levels of FMDV specific IgA become elevated transiently during the acute 
phase of infection and were stronger in FMDV carrier animals irrespective of vaccination status. Out of three mucosal fluids, nasal secretion contained the highest level of anti-FMDV IgA antibody. However, a statistically significant difference $(p \leq 0.05)$ in the anti-FMDV IgA antibody response was found between the three fluids only on $35 \mathrm{dpc}(p=0.03), 42 \mathrm{dpc}$ $(0.001)$ and $63 \mathrm{dpc}(p=0.04)$ by ANOVA analysis. Both nasal and saliva fluid collection from ruminants are considered as non-invasive methods, whereas collection of OPF is invasive and difficult to do. Moreover, difficulty in collecting uniform OPF samples may contribute to inconsistent virus detection during consecutive probang sampling from known carrier animals. Cattle do not like and usually resist the collection of nasal fluids from their nostrils, whereas collection of saliva is much easier. Therefore, a salivary IgA test might be taken forward in outbreak situation in the field, even though the nasal IgA test has slightly superior test characteristics.

Supplementary Materials: Supplementary materials are available online at https://www.mdpi. com/article/10.3390/v13050814/s1, Table S1: Principal outcomes of four O1 Manisa/ O UKG vaccine challenge experiments. Four O1 Manisa/O UKG vaccine challenge experiments were carried out in the bio-security containment facility; Pirbright Labrotary, U.K. Animals in each experiment were assigned with 2 letter identifiers (UV, UY, VD/VE, and VH).

Author Contributions: Conceptualization and acquisition of funding, S.P.; methodology, J.K.B. and S.P.; statistical analysis, A.D.N. and J.K.B.; supervision, S.P., D.J.P. and G.T.; writing first draft, J.K.B.; writing, review and editing, A.D.N., G.T., D.J.P. and S.P. All authors have read and agreed to the published version of the manuscript.

Funding: This work was financially supported by DEFRA (Department for environment, food and rural affairs, grant numbers SE1125, SE1127, SE1129 and SE1130), and EU FMD-DISCONVAC (project no. 226556) and Commonwealth Scholarship Commission, UK. J.K.B was the recipient of Commonwealth Scholarship. SP acknowledges funding from BBSRC (grant codes: BBS/E/I/00001703, $\mathrm{BBS} / \mathrm{E} / \mathrm{I} / 00007031$ and BBS/E/I/00007036-37). SP is a visiting professor at the Royal Veterinary College (RVC), University of London and a Jenner investigator at the Jenner Institute, Oxford University.

Institutional Review Board Statement: All the animal experiments were conducted in accordance with UK Home Office (HO) Rules and approved by The Pirbright Institute Ethics Committee. Procedures and endpoints were defined in HO Project Licenses (PPL 70/5900) and were conducted by qualified persons as specified in their Personal HO Licenses.

Acknowledgments: Authors would like to thank Sara Cox and Paul Barnet for allowing SP to collect nasal fluid, blood, OPF and saliva samples from ongoing vaccine experiments at The Pirbright Institute.

Conflicts of Interest: The authors declare no conflict of interest.

\section{References}

1. Belsham, G.J. Distinctive features of foot-and-mouth disease virus, a member of the picornavirus family; aspects of virus protein synthesis, protein processing and structure. Prog. Biophys. Mol. Biol. 1993, 60, 241-260. [CrossRef]

2. Grubman, M.J.; Baxt, B. Foot-and-Mouth Disease. Clin. Microbiol. Rev. 2004, 17, 465-493. [CrossRef]

3. Parida, S. Vaccination against foot-and-mouth disease virus: Strategies and effectiveness. Expert Rev. Vaccines 2009, 8, 347-365. [CrossRef]

4. Salt, J. The carrier state in foot and mouth disease-An immunological review. Br. Vet. J. 1993, 149, 207-223. [CrossRef]

5. Biswal, J.K.; Ranjan, R.; Subramaniam, S.; Mohapatra, J.K.; Patidar, S.; Sharma, M.K.; Bertram, M.R.; Brito, B.; Rodriguez, L.L.; Pattnaik, B.; et al. Genetic and antigenic variation of foot-and-mouth disease virus during persistent infection in naturally infected cattle and Asian buffalo in India. PLoS ONE 2019, 14, e0214832. [CrossRef]

6. Ilott, M.C.; Salt, J.S.; Gaskell, R.M.; Kitching, R.P. Dexamethasone inhibits virus production and the secretory IgA response in oesophageal-pharyngeal fluid in cattle persistently infected with foot-and-mouth disease virus. Epidemiol. Infect. 1997, 118, 181-187. [CrossRef] [PubMed]

7. Parthiban, A.B.R.; Mahapatra, M.; Gubbins, S.; Parida, S. Virus Excretion from Foot-And-Mouth Disease Virus Carrier Cattle and Their Potential Role in Causing New Outbreaks. PLoS ONE 2015, 10, e0128815. [CrossRef] [PubMed]

8. Horsington, J.; Zhang, Z. Consistent change in the B-C loop of VP2 observed in foot-and-mouth disease virus from persistently infected cattle: Implications for association with persistence. Virus Res. 2007, 125, 114-118. [CrossRef]

9. Alexandersen, S.; Zhang, Z.; Donaldson, A.I. Aspects of the persistence of foot-and-mouth disease virus in animals-The carrier problem. Microbes Infect. 2002, 4, 1099-1110. [CrossRef] 
10. Dawe, P.S.; Flanagan, F.O.; Madekurozwa, R.L.; Sorensen, K.J.; Anderson, E.C.; Foggin, C.M.; Ferris, N.P.; Knowles, N.J. Natural transmission of foot-and-mouth disease virus from African buffalo (Syncerus caffer) to cattle in a wildlife area of Zimbabwe. Vet. Rec. 1994, 134, 230-232. [CrossRef]

11. Arzt, J.; Belsham, G.J.; Lohse, L.; Bøtner, A.; Stenfeldt, C. Transmission of Foot-and-Mouth Disease from Persistently Infected Carrier Cattle to Naive Cattle via Transfer of Oropharyngeal Fluid. mSphere 2018, 3, e00365-18. [CrossRef]

12. Parida, S.; Cox, S.; Reid, S.; Hamblin, P.; Barnett, P.; Inoue, T.; Anderson, J.; Paton, D. The application of new techniques to the improved detection of persistently infected cattle after vaccination and contact exposure to foot-and-mouth disease. Vaccine 2005, 23, 5186-5195. [CrossRef]

13. Paton, D.J.; De Clercq, K.; Greiner, M.; Dekker, A.; Brocchi, E.; Bergmann, I.; Sammin, D.J.; Gubbins, S.; Parida, S. Application of non-structural protein antibody tests in substantiating freedom from foot-and-mouth disease virus infection after emergency vaccination of cattle. Vaccine 2006, 24, 6503-6512. [CrossRef]

14. Parida, S.; Anderson, J.; Cox, S.J.; Barnett, P.V.; Paton, D.J. Secretory IgA as an indicator of oro-pharyngeal foot-and-mouth disease virus replication and as a tool for post vaccination surveillance. Vaccine 2006, 24, 1107-1116. [CrossRef]

15. Brocchi, E.; Bergmann, I.; Dekker, A.; Paton, D.; Sammin, D.; Greiner, M.; Grazioli, S.; De Simone, F.; Yadin, H.; Haas, B.; et al. Comparative evaluation of six ELISAs for the detection of antibodies to the non-structural proteins of foot-and-mouth disease virus. Vaccine 2006, 24, 6966-6979. [CrossRef] [PubMed]

16. Parida, S.; Fleming, L.; Oh, Y.; Mahapatra, M.; Hamblin, P.; Gloster, J.; Paton, D. Emergency vaccination of sheep against foot-and-mouth disease: Significance and detection of subsequent sub-clinical infection. Vaccine 2008, 26, 3469-3479. [CrossRef] [PubMed]

17. Archetti, I.L.; Amadori, M.; Donn, A.; Salt, J.; Lodetti, E. Detection of foot-and-mouth disease virus-infected cattle by assessment of antibody response in oropharyngeal fluids. J. Clin. Microbiol. 1995, 33, 79-84. [CrossRef]

18. Salt, J.S.; Mulcahy, G.; Kitching, R.P. Isotype-specific antibody responses to foot-and-mouth disease virus in sera and secretions of "carrier" and "non-carrier" cattle. Epidemiol. Infect. 1996, 117, 349-360. [CrossRef] [PubMed]

19. Moonen, P.; Jacobs, L.; Crienen, A.; Dekker, A. Detection of carriers of foot-and-mouth disease virus among vaccinated cattle. Vet. Microbiol. 2004, 103, 151-160. [CrossRef] [PubMed]

20. Cox, S.; Voyce, C.; Parida, S.; Reid, S.; Hamblin, P.; Hutchings, G.; Paton, D.; Barnett, P. Effect of emergency FMD vaccine antigen payload on protection, sub-clinical infection and persistence following direct contact challenge of cattle. Vaccine 2006, 24, 3184-3190. [CrossRef]

21. Cox, S.J.; Parida, S.; Voyce, C.; Reid, S.M.; Hamblin, P.A.; Hutchings, G.; Paton, D.J.; Barnett, P.V. Further evaluation of higher potency vaccines for early protection of cattle against FMDV direct contact challenge. Vaccine 2007, 25, 7687-7695. [CrossRef]

22. Parida, S.; Oh, Y.; Reid, S.; Cox, S.; Statham, R.; Mahapatra, M.; Anderson, J.; Barnett, P.; Charleston, B.; Paton, D. Interferon- $\gamma$ production in vitro from whole blood of foot-and-mouth disease virus (FMDV) vaccinated and infected cattle after incubation with inactivated FMDV. Vaccine 2006, 24, 964-969. [CrossRef] [PubMed]

23. Parida, S.; Fleming, L.; Gibson, D.; Hamblin, P.A.; Grazioli, S.; Brocchi, E.; Paton, D.J. Bovine Serum Panel for Evaluating Foot-and-Mouth Disease Virus Nonstructural Protein Antibody Tests. J. Vet. Diagn. Investig. 2007, 19, 539-544. [CrossRef]

24. Sutmoller, P.; Gaggero, A. Foot-and mouth diseases carriers. Vet. Rec. 1965, 77, 968-969. [CrossRef] [PubMed]

25. Sørensen, K.J.; Madsen, K.G.; Madsen, E.S.; Salt, J.S.; Nqindi, J.; Mackay, D.K.J. Differentiation of infection from vaccination in foot-and-mouth disease by the detection of antibodies to the non-structural proteins 3D, 3AB and 3ABC in ELISA using antigens expressed in baculovirus. Arch. Virol. 1998, 143, 1461-1476. [CrossRef] [PubMed]

26. Chung, W.-B.; Sorensen, K.J.; Liao, P.-C.; Yang, P.-C.; Jong, M.-H. Differentiation of Foot-and-Mouth Disease Virus-Infected from Vaccinated Pigs by Enzyme-Linked Immunosorbent Assay Using Nonstructural Protein 3AB as the Antigen and Application to an Eradication Program. J. Clin. Microbiol. 2002, 40, 2843-2848. [CrossRef] [PubMed]

27. Branscum, A.J.; Gardner, I.; Johnson, W. Estimation of diagnostic-test sensitivity and specificity through Bayesian modeling. Prev. Vet. Med. 2005, 68, 145-163. [CrossRef]

28. Lunn, D.; Spiegelhalter, D.; Thomas, A.; Best, N. The BUGS project: Evolution, critique and future directions. Stat. Med. 2009, 28, 3049-3067. [CrossRef] [PubMed]

29. Anon. Council Directive 2003/85/EC on community measures for the control of foot-and-mouth disease repealing Directive 85/511/EEC and Decisions 89/531/EEC and 96/665/EEC and amending Directive 92/46/EEC. Offic. J. Eur. Union 2003, 46, L306.

30. Kitching, R.P. Identification of foot and mouth disease virus carrier and subclinically infected animals and differentiation from vaccinated animals. Rev. Sci. Tech. l'OIE 2002, 21, 531-538. [CrossRef] [PubMed]

31. Jacobson, R.H. Validation of serological assays for diagnosis of infectious diseases. Rev. Sci. Tech. l'OIE 1998, 17, 469-526. [CrossRef] [PubMed]

32. Kitching, P.; Hammond, J.; Jeggo, M.; Charleston, B.; Paton, D.; Rodriguez, L.; Heckert, R. Global FMD control—Is it an option? Vaccine 2007, 25, 5660-5664. [CrossRef] [PubMed] 
33. Francis, M.J.; Ouldridge, E.J.; Black, L. Antibody response in bovine pharyngeal fluid following foot-and-mouth disease vaccination and, or, exposure to live virus. Res. Vet. Sci. 1983, 35, 206-210. [CrossRef]

34. Lee, F.; Jong, M.-H.; Yang, D.-W. Presence of antibodies to non-structural proteins of foot-and-mouth disease virus in repeatedly vaccinated cattle. Vet. Microbiol. 2006, 115, 14-20. [CrossRef] [PubMed] 